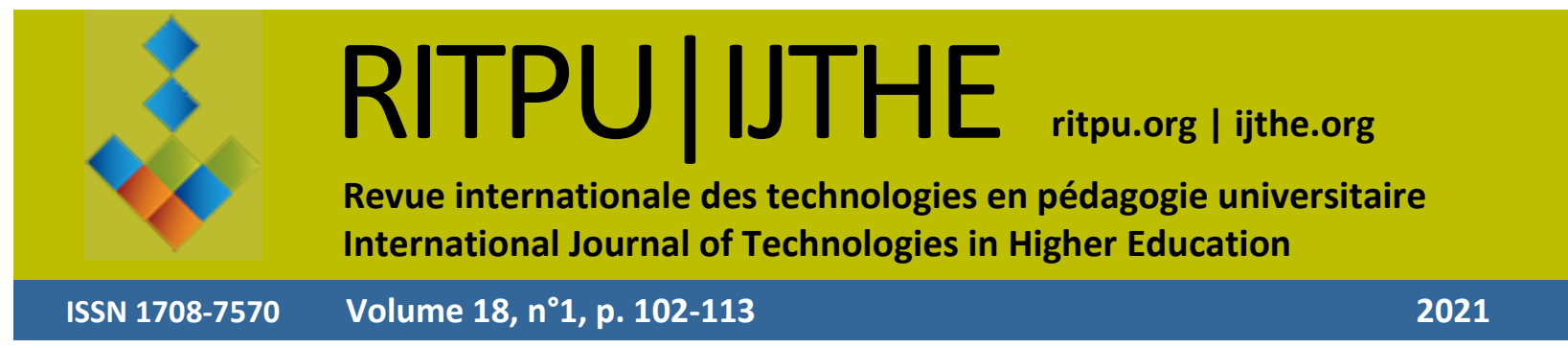

\title{
La mise en place de l'enseignement à distance au temps de la pandémie
}

Pierre-André CARON pierre-andre.caron@univ-lille.fr Laboratoire CIREL, Université de Lille

France

\section{Setting up Distance Education During the COVID-19 Pandemic}

https://doi.org/10.18162/ritpu-2021-v18n1-10

\section{Mis en ligne : 16 février 2021}

\section{Résumé}

La pandémie de COVID-19 a pour conséquence la migration en urgence des dispositifs de formation en présence vers une modalité à distance. Cet article a pour ambition de présenter quelques éléments minimaux permettant de guider cette migration. Nous commençons par situer l'enseignement à distance parmi d'autres modalités de formation, puis nous abordons la place des instruments numériques. Nous explorons alors la nature complexe du dispositif d'enseignement à distance, nous montrons qu'à une échelle micro, un tel dispositif est favorisé par la mise en place d'une activité de mobilisation et par l'organisation des apprenants en groupe.

\section{Mots-clés}

Ingénierie pédagogique, nidification, enseignement à distance, instrumentation, groupe, dispositif

\begin{abstract}
The COVID-19 pandemic has resulted in the emergency migration of face-to-face training arrangements to a distance learning modality. This article aims to present some minimal elements to guide this migration. We begin by situating distance learning among other training modalities, and then we discuss the place of digital tools. We then explore the complex nature of the distance learning system, showing that, on a micro scale, such a system is favoured by the implementation of a mobilization activity and by the organization of learners in groups.
\end{abstract}

\section{Keywords}

Pedagogical engineering, nesting, distance learning, instrumentation, group, learning device 


\section{Introduction}

La crise engendrée par la COVID-19 a amené des millions d'enseignants et de formateurs à adapter leurs cours et leurs formations. En très peu de temps, ceux-ci ont dû trouver des solutions afin de diffuser de la matière, faire réaliser des exercices et accompagner leurs étudiants à distance. Bien que certains d'entre eux explorent depuis longtemps l'usage des objets numériques, l'adaptation à cette situation reste très compliquée. Plus qu'une simple transposition des pratiques en présence, il s'agit de mettre en place une ingénierie à laquelle les enseignants et les formateurs ne sont ni formés ni aguerris. Comme beaucoup de nos collègues, nous sommes ainsi interpellés pour accompagner et guider ces enseignants dans cette ingénierie ${ }^{1}$. Nous intervenons alors dans cet accompagnement à plusieurs titres :

- En tant qu'enseignant et directeur des études d'un master décliné sous plusieurs modalités (en présence, hybride et à distance), nous sommes nous-mêmes confronté, comme tous nos collègues, à la demande institutionnelle de mettre à distance en urgence tous nos enseignements pour faciliter la continuité pédagogique.

- En tant que chercheur en sciences de l'éducation, nous étudions les difficultés spécifiques et systémiques que rencontrent nos collègues enseignants dans cette tâche de réingénierie. Cette étude trouve également sa place dans le cadre du projet de recherche européen Teach Transition, qui a pour objectif la compréhension des démarches d'ingénierie pédagogique et la formation des enseignants aux compétences numériques.

Par ailleurs, nos compétences en ingénierie pédagogique sont également fréquemment sollicitées pour le design de dispositifs de formation à distance. C'est donc sous ces trois identités d'enseignant, de chercheur et d'ingénieur pédagogique que nous avons décidé d'aborder cet article qui a pour ambition de guider les enseignants dans la mise en place de l'enseignement à distance.

Traditionnellement en formation, l'enseignement à distance est organisé selon plusieurs modèles :

- Un premier modèle, artisanal, pragmatique - Ce modèle est souvent présent dans des départements ou établissements assez polyvalents. Il s'appuie sur une forme de bricolage enseignant (Baltazart et Chagnoux, 2017; Büscher et al., 2001; Caron et al., 2010b; Perrenoud, 1983). Peraya et Peltier (2020, p. 10) précisent que dans le cadre de cet artisanat, «les enseignants tentent de projeter, sur les nouveaux dispositifs d'instrumentation de la formation, des comportements et des pratiques liés à l'enseignement présentiel frontal». Ce modèle souvent critiqué est cependant suffisamment présent en université pour que les mêmes auteurs clôturent leur proposition de débat en proposant la question suivante : «Le passage de l'artisanat à la standardisation qui constitue l'un des fondements de l'ingénierie pédagogique s'est-il réalisé dans les universités?»

- Un deuxième modèle, ingénierique - Ce modèle est principalement présent dans des services d'appui ou établissements dont la principale activité est l'enseignement à distance. Il repose sur la standardisation et la rationalisation des méthodes (Basque et al., 2010; Béziat et Wallet, 2007; Henri, 2019; Moeglin, 2010). Il théorise et développe une ingénierie pédagogique, capable de traiter « de manière rigoureuse la conception, le développement et

1. Webinaire, Université de Lille, France ([Martin T], 2020, 9:46); webinaire, Organisation internationale de la Francophonie (OIF), Sénégal (IFEF, 2020). 
la diffusion d'environnements d'apprentissage basés sur les technologies. Garante d'efficacité et de fiabilité, elle [l'ingénierie] organise et structure les diverses composantes en interaction dans les environnements et formule des prescriptions relatives à l'appropriation de connaissances et à l'acquisition de compétences » (Henri, 2019, p. 7). Ce modèle est actuellement en évolution, dans la mesure où, de plus en plus, il lui faut privilégier une focalisation sur l'accompagnement pédagogique et les dispositions des apprenants plutôt qu'exclusivement sur la médiatisation de contenu. «Face à cette masse de contenus, apprendre devient plus que jamais un acte d'orientation, de choix, de lien qui oblige chacun à se positionner» (Cristol, 2019, p. 2). Cette évolution est cependant fragilisée par la généralisation de l'usage des environnements personnels d'apprentissage. L'ingénierie pédagogique fait alors face à une situation paradoxale où il semble difficile de « concilier la rigueur prescriptive de l'ingénierie pédagogique avec la nécessité de s'ajuster aux spécificités des apprenants et à leurs usages » (Peraya et Peltier, 2020, p. 20).

C'est donc dans ce contexte de confrontation, d'évolution et de fragilisation des modèles que survient la crise de la COVID-19, crise accompagnée en éducation d'une injonction à agir et d'une sollicitation permanente concernant l'accompagnement par les pairs, par la recherche et l'ingénierie d'une aide à la migration distante des dispositifs de formation.

Cet article a ainsi plusieurs objectifs, le premier étant d'exposer quelques principes simples d'ingénierie pratiquée en enseignement à distance pour guider les enseignants dans l'appropriation de ces nouvelles pratiques. Le deuxième est de permettre à ces derniers de se familiariser avec les concepts fréquemment convoqués pour construire ces principes. Il s'agit alors pour eux d'acquérir une épaisseur théorique dans le but d'anticiper ainsi d'autres conduites. Enfin, nous craignons également que les dispositifs individuels, ou semi-collectifs, improvisés dans l'urgence, fassent courir le risque d'un discrédit durable auquel l'enseignement à distance devra faire face dans l'avenir, voire d'une validation de la thèse d'un enseignement à distance inadapté à certaines tranches d'âge ou à certains profils d'élèves. Notre contribution, à travers ces deux objectifs d'exemplarité et de théorisation, a ainsi pour but de participer à ce débat.

\section{L'enseignement à distance n'est pas un choix, pour beaucoup c'est une nécessité}

La crise actuelle et son confinement nous rappellent cette notion essentielle propre à l'enseignement à distance qui, depuis plus d'un siècle, a permis à des personnes qui n'avaient pas la possibilité de suivre un enseignement en présence d'apprendre. Cette préoccupation légitime est déjà perceptible à travers les imaginaires romancés de la fin du $19^{\mathrm{e}}$ siècle. Ainsi, dans le roman La vie électrique (Robida, 1892), Estelle Lacombe suit, par l'intermédiaire du « téléphonoscope », des cours à distance pour devenir ingénieure. Cet enseignement sous forme de visioconférence et lecture de livres lui permet non seulement de s'affranchir de la distance et de l'interdiction de quitter son foyer, mais aussi d'accéder à des connaissances interdites aux femmes. Plus récemment, la mise en place de l'enseignement à distance a permis aux établissements d'enseignement de s'affranchir de la géographie ou de la densité trop faible de population (McGreal et Anderson, 2007; Stacey et Visser, 2005), de la surpopulation étudiante (Karsenti et Collin, 2010) ou des conditions socioéconomiques de certaines régions de France et d'Europe (Albero et Thibault, 2006). Actuellement, en formation d'adulte, l'enseignement à distance permet aux adultes en recherche d'emploi, de réorientation ou d'évolution professionnelle d'accéder à un enseignement tout en conservant leur emploi. Dans ce contexte, l'enseignement à distance est en cours de généralisation et devient pour beaucoup d'apprenants la nouvelle norme pour se former. La crise actuelle touche tous les types de formation et tous les 
niveaux; elle nous rappelle que les apprenants, quels que soient leur âge ou leur statut, n'ont pas le choix. Pour eux, l'alternative est simple : apprendre ou ne pas apprendre. Il ne s'agit donc pas, dans cet article, de débattre sur les qualités de l'enseignement distant en les comparant à celles de l'enseignement en présence, mais sur la possibilité de mettre en place un enseignement à distance. Pour beaucoup, particulièrement en ces temps de confinement, l'enseignement et l'apprentissage à distance ne relèvent pas d'un choix mais d'une nécessité.

\section{Apprendre à distance}

L'enseignement à distance instaure une séparation entre planification des enseignements et apprentissages :

Dans la formation à distance, l'apprenant utilise, souvent seul, des ressources éducatives sur différents supports dans un lieu qui n'est pas une salle de cours. Ce lieu peut être son domicile, l'entreprise où il travaille, un centre de ressources, etc. Les ressources éducatives ont été produites antérieurement par des enseignants ou des spécialistes qui sont alors généralement absents de ce lieu. La formation à distance implique donc, par définition, une séparation dans l'espace et le temps des activités d'enseignement et d'apprentissage. (Glikman, 2002, p. 12)

Pour (Peraya et Peltier, 2020), le fait de distinguer, dans les formations à distance, enseignement et apprentissage justifie la place importante que prennent, dans ces formations, l'ingénierie pédagogique " traditionnelle » et la séparation des rôles qu'elle instruit. Les auteurs précisent que ce modèle d'ingénierie comporte alors deux aspects fondamentaux, la nécessité de planifier et celle de médiatiser. Un tel modèle ingénierique, comme nous le rappelions en introduction, n'est cependant pas réellement accessible aux enseignants qui vivent actuellement une injonction institutionnelle, professionnelle et déontologique qui ne s'est accompagnée d'aucune formation. Le risque est alors grand de les voir procéder à une simple transposition des pratiques antérieures (Peltier, 2020). Selon Perrenoud (1998), la transposition des pratiques professionnelles fait parfois perdre de vue les activités de référence et les compétences que nous cherchons à construire, comme en témoigne Caron (2020a).

J'ai ainsi l'exemple de ces enseignants : - qui distribuent des PDF à leurs élèves de collège et leur demandent de les recopier sur leur cahier pour les mémoriser plus facilement; - qui s'enregistrent pendant quatre heures et qui diffusent sur les réseaux ce que précédemment ils animaient en amphi, sans savoir s'ils sont regardés et par qui; - qui, dans le secondaire, convoquent à heure fixe des élèves pour des visio-conférences synchrones de plus d'une heure et qui s'étonnent de ne mobiliser que le dixième de leur classe, tout en concluant [à] l'impossibilité d'enseigner à distance sans augmenter l'inégalité scolaire. (p. 4)

Pour l'enseignant, penser ses pratiques professionnelles autrement, c'est ne plus organiser son enseignement autour de la délivrance et de l'explicitation collective et synchrone d'éléments de cours s'articulant ou non avec des activités de découverte, d'entraînement, d'approfondissement ou d'évaluation. Il s'agit pour lui de procéder à une inversion de perspective : ne plus se demander s'il est possible d'enseigner et de transposer ses pratiques, mais s'il est possible pour les élèves d'apprendre à distance. Or plus d'un siècle d'enseignement à distance nous le prouve, il est possible d'apprendre à distance pour peu que l'ingénierie mise en place le favorise. Il est possible d'apprendre à distance si, en tant qu'enseignants, nous acceptons de penser notre enseignement autrement : non par rapport à des pratiques à transposer, mais par rapport à de 
nouvelles pratiques à mettre en place pour que l'apprenant atteigne les objectifs que nous nous sommes donnés en matière de savoirs et de compétences.

En enseignement à distance, la flexibilité et la dilatation du temps (Glikman, 2002, p. 12), ainsi que la mise en réseau des apprenants et des enseignants, font de la planification des activités et des interactions qu'elles sont susceptibles de susciter un des éléments fondamentaux de l'ingénierie que l'enseignant doit développer.

D’une part, la réalisation d'activités permet, selon Piaget (1975), l'évolution de la structure cognitive de l'apprenant par réorganisation de schèmes, à la suite de déséquilibres suivis de rééquilibrations majorantes.

D'autre part, lors de la réalisation de ces activités, les interactions avec l'enseignant ou entre les pairs permettent la mise en place de conflits sociocognitifs qui favorisent alors le développement cognitif de l'apprenant (Vygotski, 1934/1985).

Nous proposons dans le cadre de cet article une planification assez simple reposant sur la mise en place d'activités et les interactions qu'elles génèrent. L'étudiant est mobilisé, puis il se voit proposer des activités. La réalisation de ces activités nécessite pour lui l'explicitation des consignes, l'interaction et la coordination avec les autres et la lecture du cours mis ou non à sa disposition. Il rend son travail et il lit la correction. L'enseignant scénarise son cours autour de plusieurs activités que va devoir réaliser l'étudiant. Il mobilise ses étudiants puis leur propose des activités, il écrit ou désigne un cours qu'il met à la disposition des étudiants. Il répond aux sollicitations, organise le travail de groupe. Il corrige et évalue les activités.

Donc, en résumé, voici les étapes schématiques d'un cours à distance telles que nous les proposons : mobilisation et activités entraînent interactions et appropriation du cours, elles donnent lieu à un rendu qui est évalué. La mobilisation initiale des étudiants est entretenue par la proposition d'activités qui seront corrigées et évaluées. Les activités permettent aux apprenants de tester leurs savoirs et leurs compétences, elles justifient la lecture du cours, la recherche d'informations et les échanges entre pairs ou avec l'enseignant. Le rendu du travail permet l'institutionnalisation scolaire d'un travail personnel, ou de groupe, réalisé au sein de la sphère privée. En enseignement à distance, comme la notion de lieu est virtuelle, ce changement de statut, cette institutionnalisation du travail réalisé, ne compte pas parmi les caractéristiques du dispositif; c'est donc une étape primordiale qu'il faut instrumenter dans la mesure où elle participe de la reconnaissance du savoir et des compétences acquises. La correction et l'évaluation achèvent cette reconnaissance et concourent à entretenir la mobilisation des apprenants.

\section{L'usage des outils numériques}

L'enseignement à distance est caractérisé par l'importance apportée aux outils et aux services de médiation, autrefois le service postal, la radio, la télévision, aujourd'hui Internet et les applications qui utilisent le réseau (Glikman, 2002). Cependant, en cette période d'urgence, les enseignants et les apprenants n'ont pas la possibilité temporelle de devenir des professionnels de ces outils de médiation. La place des outils, dans l'ingénierie de l'enseignant, concerne donc, d'une part, le choix et l'instrumentation d'outils simples et malléables permettant à l'enseignant de déployer son scénario et, d'autre part, le fait de favoriser l'autonomie instrumentale des apprenants. Or la recherche de l'outil parfait, réduit à ses fonctionnalités déclarées ou juste perçues, focalise l'enseignant sur les aspects techniques au détriment d'une réflexion sur les pratiques qu'il désire instrumenter. Nous suggérons une focalisation non pas sur l'outil et ses 
fonctionnalités supposées, déclarées ou perçues, mais sur les pratiques que chaque enseignant souhaite mettre en place pour instrumenter les étapes décrites précédemment : la mobilisation des apprenants, les interactions entre pairs et avec l'enseignant, le rendu de devoir et de corrections, l'exposition d'activités et d'éléments de cours. En matière d'espaces, il s'agit donc de construire :

- Un espace de mobilisation (courriel, Skype, Slack, Discord, WhatsApp, etc.), toutes ces applications permettant de mobiliser en notifiant. Cet espace peut être couplé avec un lieu d'échange synchrone (tablette synchrone, classe virtuelle, etc.);

- Un espace de dialogue asynchrone (de type forum consultable et organisable). Les applications précédentes peuvent être utilisées et détournées, les plateformes de formation classiques également. Il est à ce stade possible de distinguer le lieu scolaire de dialogue avec l'enseignant et le lieu éventuellement privé de dialogue entre pairs;

- Un espace de rendu des devoirs et des corrections (partage infonuagique d'un répertoire, lieu de rendu sur une plateforme de formation, etc.);

- Un espace d'exposition du cours et des activités (partage infonuagique d'un répertoire, espace d'exposition d'une plateforme, site Web, blogue).

La mise en place de ces différents espaces peut être l'occasion de mobiliser différents outils et services, mais il ne faut pas négliger l'importance de la genèse instrumentale qui, couplée avec la genèse professionnelle de l'enseignant et de l'apprenant, permettra la mise en place progressive des schèmes d'usage. Il est possible, d'une part, de privilégier dans un premier temps des outils simples, malléables, accessibles et facilement appropriables par leurs usagers (enseignants et apprenants) (Caron et Varga, 2009) et, d'autre part, de faire en sorte que l'ensemble de la communauté éducative utilise les mêmes outils; cela permettra de favoriser leur instrumentation pour l'apprenant.

\section{Le collectif éducatif et le dispositif}

Un des enseignements principaux de cette crise, au-delà des difficultés individuelles que rencontre chaque enseignant lors de la construction et de l'animation de ses cours, est la difficulté à mobiliser le collectif autour d'un nouveau dispositif de formation intégrant le distant. Or c'est ce collectif qui, à travers l'ingénierie qu'il porte, est chargé de prescrire et d'accompagner la mise en place d'un enseignement, qu'il soit en présence ou à distance. Classiquement, pour l'enseignement en présence, le collectif éducatif édicte et suit des règles qui sont littéralement inscrites dans le dispositif pédagogique complexe et concret au sein duquel l'enseignement en présence se déploie. Le dispositif est ainsi concrétisé (réifié) par de multiples éléments prescriptifs réels et structurels (établissement scolaire, cours de récréation, programme des cours, règlement de l'établissement, livres, cahiers, taille des cahiers, interlignes, couleur des stylos, salles, organisation topologique des salles, classes, promotions, regroupement, agenda, organisation des créneaux horaires, sonnerie, différents rôles au sein de l'établissement, etc.). En un sens, le dispositif réel inscrit incarne et prescrit les règles de fonctionnement de ses usagers et permet la professionnalisation des nouveaux enseignants et des nouveaux étudiants ou élèves de même que la résilience du système de formation en cas de modification de quelques paramètres. En enseignement à distance, ce même collectif trouve également des règles de fonctionnement, de prescription et de résilience dans un dispositif à construire qui est différent car en partie virtuel. Bien que virtuel, ce dispositif est sans doute tout autant prescriptif (Caron, 2013, 2015; Caron, Charlier et Peraya, 2018). La proposition que nous faisons s'inscrit dans une logique 
d'ingénierie minimaliste et développe une forme toute particulière de prescriptibilité, en l'intégrant à un dispositif commun proposé aux enseignants par l'établissement. Les caractéristiques de sédimentation et de panoptique propres aux instruments numériques permettent alors, comme nous l'avons montré depuis plusieurs années, de prescrire et de cristalliser les pratiques pédagogiques des enseignants et des apprenants (Caron et al., 2010a). Dans la situation actuelle, nous suggérons que c'est sans doute la non-construction de ce dispositif commun, en partie virtuel, ainsi que le manque de consensus autour de ce dispositif qui sont à l'origine des difficultés singulières que rencontre chaque enseignant. En cette période, il s'agit donc moins pour l'établissement de former à enseigner à distance que d'offrir une offre de signification qui permette au collectif de structurer ses actions, en produisant du sens pour tous. Dans ce dispositif à la construction inachevée, l'enseignant en partie livré à lui-même cherche dans la transposition simple de son propre micro-dispositif habituel, les règles pour prescrire ses actions et celles de ses élèves. Cet article est donc aussi là pour lui indiquer les éléments facilement transposables et ceux qu'il lui faut construire en priorité. Chacun doit cependant comprendre que son action individuelle s'inscrit dans un dispositif plus large, en cours de formalisation par son établissement, voire par son institution. En cette période d'urgence, l'enseignant, par ses actions, ne peut guère agir que sur la dimension micro du dispositif, en structurant les échanges, en formalisant les activités et le cours, en fixant les échéances et les travaux à rendre et en prescrivant les aspects instrumentaux, des éléments qui ont tous été abordés dans notre article. Enfin, il est également possible à l'enseignant d'agir par subsidiarité au collectif, dans le cadre de ses actions, sur l'isolement des apprenants.

\section{Construire le groupe}

Pour agir sur cet isolement, une première étape que l'enseignant peut mettre en œuvre en est une de mobilisation telle que nous l'avons décrite précédemment. Classiquement, en enseignement à distance, cette étape n'est pas prise en charge au niveau d'un cours mais plutôt d'une promotion. Cette mobilisation nécessite concrètement de pouvoir joindre les élèves (par courriel ou téléphone intelligent). Elle consiste à créer un évènement de rentrée synchrone débutant par plusieurs activités simples à réaliser dans le but, par exemple, d'aider les apprenants à construire leur identité numérique et à apprivoiser l'environnement instrumental. Cette mobilisation permet de favoriser, par le stress du synchrone, le repérage et l'organisation physique et sociale de leur espace personnel d'apprentissage.

Même si le basculement entre enseignement en présence et enseignement à distance se déroule en cours d'année, il est sans doute nécessaire de mettre en place cet évènement de mobilisation et d'introduction au distant. En particulier, il faut prendre conscience que les apprenants ont construit une identité en présence qui est en partie distincte de celle qu'ils construiront numériquement. En enseignement en présence, cette identité s'est construite dans des lieux neutres - la salle de classe, la cour, le chemin de l'établissement scolaire; en enseignement à distance, elle se construit paradoxalement dans des lieux plus intimes. Cette construction identitaire doit donc être introduite graduellement avec précaution.

Dans nos formations à distance, nous privilégions pour soutenir la volition, une forme d'appariement (Bretherton, 1992) que nous nommons nidification (Caron et Androwkha, 2019), cette nidification assistée permet de minimiser le stress pendant la première phase de formation, elle facilite l'établissement d'un premier groupe de travail stable (Institut de la francophonie pour l'éducation et la formation [IFEF], 2020). 
Nous avons l'exemple d'autres formations qui ont recours, pour répondre au même objectif, à une mallette d'objets, «Une boîte à outils a été développée selon une approche itérative de type « through design ». Cinq objets tangibles sont proposés pour répondre de façon personnalisée aux besoins des étudiants en termes de stratégies » (Molinari et Schneider, 2020, p. 17).

Une fois le groupe créé, il s'agit alors de lui proposer des activités. En effet, contrairement à l'enseignement en présence, l'enseignement à distance instaure un dialogue continuel entre l'enseignant et l'apprenant (explicitation des consignes, approfondissement épistémique); cette multiplication d'interactions privées est chronophage.

En formation à distance nous exigeons, quand un étudiant adresse à l'enseignant une question, que cette question, et la réponse donnée, soient publiques (forum asynchrone), nous demandons aussi aux enseignants de répondre dans un délai de trois jours, mais de ne pas répondre non plus immédiatement, pour laisser aux autres étudiants la possibilité de répondre ou d'approfondir la question. Nous alertons également les enseignants vis-à-vis du fait d'être trop précis dans les consignes données, cette précision est paradoxalement en enseignement à distance contre-productive, cela tient à plusieurs points :

- Le premier concerne le fait que les échanges sont écrits, et donc se sédimentent, il y a alors une dérive naturelle d'année en année vers une précision de plus en plus grande qui alourdit pour l'étudiant la tâche à réaliser;

- Le deuxième tient au fait que l'explicitation des consignes est un bon déclencheur des interactions entre pairs dont il serait dommage de se priver;

- Enfin le dernier est plus subtil, il tient à la distinction que tout enseignant doit faire entre la tâche à réaliser et la compétence à construire. Nos étudiants, issus du monde du travail, sont habitués à réaliser au mieux les tâches demandées lorsque les consignes sont explicites, ils ont donc des difficultés à comprendre qu'en formation la tâche n'est qu'un prétexte à la construction des compétences. (IFEF, 2020)

Concernant cette distinction entre tâche et compétence et en particulier sur la responsabilité de l'enseignant, il faut noter que cette problématique n'est pas la caractéristique du distant, c'est plutôt qu'en enseignement à distance, toutes les interactions laissent des traces dans le système d'information, tout est explicite... même le fait de ne pas l'être.

Le sujet ne doit plus placer sa satisfaction dans la réalisation de la tâche mais dans le progrès que le franchissement de l'obstacle lui permettra de faire [...] Le rôle du maître est donc ici essentiel : il doit, en quelque sorte, empêcher que l'élève puisse « réussir » sans « comprendre ». (Meirieu, 2005, p. 50)

Enfin, il est important de prendre en compte les étudiants qui ne posent pas de questions, mais qui peuvent se reconnaître dans les questions posées par les autres. Dans la littérature anglosaxonne, ils sont désignés par le terme «lurkers »; ils bénéficient des interactions des autres étudiants sans y participer. Or dans une communauté d'apprentissage, communauté intentionnelle construite institutionnellement autour d'un projet commun d'apprentissage, les «lurkers» occupent une place assez bien identifiée, celle de la participation légitime périphérique (Lave, 1991), apprenant par leur observation des interactions des autres. Cette participation légitime périphérique leur permet de mettre en œuvre un apprentissage vicariant (Bandura, 1976). En enseignement classique et en formation d'adulte à fortiori, en utilisant les 
moyens numériques et leurs propriétés panoptiques, cet apprentissage vicariant est une modalité très commune d'apprentissage qu'il s'agit de ne pas négliger (Vieille-Grosjean et Di Patrizio, 2015).

Une fois l'ingénierie dispositive déployée, à travers ses scénarios simples, ses choix instrumentaux, ses constructions identitaires, ses regroupements et nidifications, et ses règles de conduite, le dispositif génère naturellement des interactions entre les différents acteurs. Ces interactions sont assez stables et ne dépendent pas réellement du nombre d'étudiants regroupés. «Dans notre master, que nous ayons 12 étudiants, 25, 35 voire 45 dans une promo, le dispositif génère chaque année environ 6000 messages, ce nombre est depuis plus de 15 ans identique, stable et indépendant du nombre d'étudiants » (IFEF, 2020). Pour gérer pédagogiquement ce nombre assez stable mais cependant important d'interactions, une des solutions pédagogiques constamment préconisées pour éviter la mise en place de dialogue chronophage consiste à augmenter les travaux de groupe pour substituer à l'expertise de l'enseignant une expertise par les pairs (Béziat et Wallet, 2007; Deceuninck, 2005; D'Halluin, 2001; Poellhuber et al., 2012; Saint-Arnaud, 1989). Or travailler en groupe signifie construire dans un premier temps une identité et des compétences individuelles. Le travail de groupe implique donc, pour l'enseignant, de construire l'interdépendance positive entre les apprenants d'un même groupe (Johnson et Johnson, 1987). Cette interdépendance peut par exemple se construire par des consignes simples (tous les membres du groupe auront la même note) ou par des motifs pédagogiques plus élaborés de type «jigsaw » (Aronson et Patnoe, 1997).

\section{Conclusion}

Dans cet article, nous avons souhaité aborder la mise en place pour un enseignant d'un dispositif de formation à distance et les règles simples qui en régissent l'usage. Nous avons présenté l'articulation qui existe entre les différentes étapes d'un cours : la mobilisation des étudiants, la réalisation des activités, les interactions qu'elles suscitent entre les différents acteurs, la lecture du cours qu'elles génèrent et l'évaluation de ces activités par l'enseignant. Pour instrumenter ces étapes, nous avons décrit les différents espaces numériques que l'enseignant et les apprenants peuvent investir et instrumenter. Enfin, nous avons montré la nécessité de penser, au niveau du collectif éducatif, le dispositif complexe à mettre en place. Au sein de ce dispositif, et particulièrement au cours de cette période de crise, l'enseignant ne peut guère agir qu'au niveau micro. Il peut toutefois mettre en place des travaux de groupe lui permettant, d'une part, d'éviter des activités trop chronophages et, d'autre part, de construire au sein de son cours une communauté d'apprentissage capable de donner du sens au projet commun d'enseignement et d'apprentissage.

Cet article n'a pas d'autres ambitions que de donner, en cette période particulière où urgence rime avec mutation pédagogique et instrumentale, quelques clefs d'une ingénierie minimaliste et contemporaine. Une de ses limites concerne assurément la pluralité des modalités de formation en présence. Si les quelques pratiques présentées dans cet article s'appliquent sans doute assez bien à un enseignement classique de type artisanal, on perçoit bien la difficulté de transposer ces pratiques dans des modalités d'enseignement au plus grand nombre (par exemple dans les premières années du cycle universitaire).

À travers cette proposition, nous espérons avoir permis la compréhension des mécanismes qui régissent un dispositif de formation à distance. Pour ce faire, nous avons tenté d'articuler plusieurs discours, un premier, pragmatique, pour tenter de livrer le plus simplement possible ces clefs d'ingénierie et un deuxième plus réflexif permettant d'exposer les justifications théoriques 
de ces prescriptions, auxquels s'ajoute notre choix d'illustrer cet article d'extraits d'un témoignage que nous avons réalisé sous forme de webinaire pour l'Organisation internationale de la Francophonie (OIF) (IFEF, 2020). Nous remercions à ce titre l'OIF, pour la possibilité qu'elle nous a offerte de nous exprimer sur ce sujet.

\section{Références}

Albero, B. et Thibault, F. (2006). ELUE France. Dans F. Thibault, B. Albero, P. Kess, P. Tolonen, H. Salovaara, C. Rita Alfonsi, M. Carfagna et D. Marsiglia (dir.). CRUI, CPU, FVU : les universités européennes à l'heure du e-learning : regard sur la Finlande, l'Italie et la France (p. 61-98). Conférence des présidents d'universités italiennes (CRUI). http://edutice.archives-ouvertes.fr/...

Aronson, E. et Patnoe, S. (1997). The jigsaw classroom: Building cooperation in the classroom ( $2^{\mathrm{e}}$ éd.). Allyn \& Bacon.

Baltazart, D. et Chagnoux, M. (2017). Écologie numérique des enseignants-chercheurs : entre habitus pédagogique et bricolage personnel. Dans L. Massou et N. Lavielle-Gutnik (dir.), Enseigner à l'université avec le numérique (p. 43-64). De Boeck Supérieur.

Bandura, A. (1976). L'apprentissage social [Social learning theory] (J. A. Rondal, trad.). Pierre Mardaga.

Basque, J., Contamines, J. et Maina, M. (2010). Approches de design des environnements d'apprentissage. Dans B. Charlier et F. Henri (dir.), Apprendre avec les technologies (p. 109-119). Presses universitaires de France.

Béziat, J. et Wallet, J. (2007). L'étudiant et la distance. Dispositif de formation et pratiques sociales. Dans J. Wallet (dir.), Le campus numérique FORSE : analyses et témoignages (p. 65-76). Universités de Rouen et du Havre.

Bretherton, I. (1992). The origins of attachment theory: John Bowlby and Mary Ainsworth. Developmental Psychology, 28(5), 759-775. https://doi.org/10.1037/0012-1649.28.5.759

Büscher, M., Gill, S., Mogensen, P. et Shapiro, D. (2001). Landscapes of practice: Bricolage as a method for situated design. Computer Supported Cooperative Work, 10(1), 1-28. https://doi.org/10.1023/A:1011293210539

Caron, P.-A. (2013). Proposition de cadres théoriques propres aux TICE à partir de recherches liées au champ de la professionnalisation. Dans M. Cantisano et D. Groux (dir.), Professionnalisation et e-learning (p. 67-77). L'Harmattan.

Caron, P.-A. (2015). Usage des TICE pour soutenir les enjeux de la professionnalisation des enseignants à l'université. Dans L. Roger (dir.), Mise en auvre de la professionnalisation dans l'enseignement supérieur (p. 80-100). Champ social.

Caron, P.-A. (2020a). Ingénierie dispositive et enseignement à distance au temps de la COVID-19. Distances et médiations des savoirs, (30). https://doi.org/10.4000/dms.5211

Caron, P.-A. et Androwkha, S. (2019). Nidification dans un dispositif de formation à distance pour adulte. Dans Pré-actes du colloque Éducation 4.1 - Distances, médiations des savoirs et des formations (p. 176-191). http://education4-1.sciencesconf.org/... 
Caron, P.-A., Becerril Ortega, R. et Rethoré, S. (2010a). Analyse quantitative des traces d'activités sur un artefact dédié aux échanges pédagogiques. Lien entre instrumentation et usage. Dans Actes du $7^{e}$ colloque Technologies de l'information et de la communication pour l'enseignement (TICE2010). http://halshs.archives-ouvertes.fr/...

Caron, P.-A., Becerril Ortega, R. et Rethoré, S. (2010b). Modèle artisanal de la formation à distance, lien entre l'artefact et la pédagogie. Dans M. Sidir, É. Bruillard et G.-L. Baron (dir.), Actes des journées Communication et apprentissage instrumentés en réseau (Jocair 10) (p. 10-23). INRP.

Caron, P.-A., Charlier, B. et Peraya, D. (2018, 23 mars). Table ronde : l'usage d'un dispositif instrumenté est-il perçu par les formateurs l'utilisant comme une forme de professionnalisation ? [vidéo]. $2^{\text {ème }}$ colloque international e-Formation des adultes et des jeunes adultes. Webtv. https://webtv.univ-lille.fr/...

Caron, P.-A. et Varga, R. (2009). Artefacts malléables et perméables (AMP) pour mener des activités pédagogiques : l'usager acteur et créateur de son dispositif numérique. Distances et savoirs, 7(2), 155-177. https://doi.org/10.3166/ds.7.155-177

Cristol, D. (2019, 25 février). Bouleversements pédagogiques. Thot Cursus. http://cursus.edu/...

Deceuninck, J. (2005). Campus Forse, formation et ressources en sciences de l'éducation [rapport exploratoire]. Dans Présentation des résultats 2005 de l'ERTe. ERTe Modèles économiques et enjeux organisationnels des campus numériques. http://erte.mshparisnord.org/...

D’Halluin, C. (dir.). (2001). Usages d'un environnement médiatisé pour l'apprentissage coopératif [numéro thématique]. Cahiers d'études du centre université-économie d'éducation permanente (CUEEP), (43). http://transformations.univ-lille.fr/...

Glikman, V. (2002). Des cours par correspondance au e-learning. Presses universitaires de France.

Henri, F. (2019). Quel changement à l'ère numérique? Quelle ingénierie pédagogique pour y répondre? Médiations et médiatisations, (2), 227-235.

http://revue-mediations.teluq.ca/...

Institut de la francophonie pour l'éducation et la formation [IFEF OIF]. (2020, 8 avril). Vademecum, enseignement à distance (webinaire IFEF) [vidéo]. YouTube. http://youtu.be/...

Johnson, D. W. et Johnson, R. T. (1987). Learning together and alone: Cooperative, competitive, and individualistic learning. Prentice-Hall.

Karsenti, T. et Collin, S. (2010). Les formations ouvertes à distance (FOAD) : quelle contribution au développement de professionnels qualifiés en Afrique? Questions vives, recherches en éducation, 7(14), 71-87. https://doi.org/10.4000/questionsvives.536

Lave, J. (1991). Acquisition des savoirs et pratiques de groupe. Sociologie et sociétés, 23(1), 145-162. https://doi.org/10.7202/001418ar

[Martin T]. (2020, 26 mars). Échange de pratiques du CAPE : enseignement à distance avec Pierre-André Caron [vidéo]. YouTube. http://youtu.be/...

McGreal, R. et Anderson, T. (2007). E-learning in Canada. International Journal of Distance Education Technologies, 5(1), 1-6. https://doi.org/10.4018/jdet.2007010101 
Meirieu, P. (2005). Si la compétence n'existait pas, il faudrait l'inventer. Site de l'auteur. http://meirieu.com/...

Moeglin, P. (2010). Les industries éducatives. Presses universitaires de France.

Molinari, G. et Schneider, E. (2020). Soutenir les stratégies volitionnelles et améliorer l'expérience des étudiants en formation à distance. Quels potentiels pour le design tangible? Distances et médiations des savoirs. Distance and Mediation of Knowledge, (32). https://doi.org/10.4000/dms.5731

Peltier, C. (2020, $1^{\mathrm{er}}$ avril). INFOX\#7 : Le e-learning n'est qu'une simple transposition d'un cours présentiel. Laboratoire d'innovation pédagogique, CERF, Université de Fribourg. http://lip-unifr.ch/...

Peraya, D. et Peltier, C. (2020). Ingénierie pédagogique : vingt fois sur le métier remettons notre ouvrage... Distances et médiations des savoirs, (29). https://doi.org/10.4000/dms.4817

Perrenoud, P. (1983). La pratique pédagogique entre l'improvisation réglée et le bricolage Essai sur les effets indirects de la recherche en éducation. Éducation \& Recherche, 5(2), 198-212. http://e-periodica.ch/...

Perrenoud, P. (1998). La transposition didactique à partir de pratiques : des savoirs aux compétences. Revue des sciences de l'éducation, 24(3), 487-514. https://doi.org/10.7202/031969ar

Poellhuber, B., Roy, N., Racette, N. et Anderson, T. (2012). Le profil technologique des étudiants canadiens en formation à distance et les déterminants de leur intérêt envers la collaboration et l'utilisation des logiciels sociaux. Dans T. Karsenti et S. Collin (dir.), TIC, technologies émergentes et Web 2.0 : quels impacts en éducation? (p. 310-369). CRIFPE.

Piaget, J. (1975). L'équilibration des structures cognitives : problème central du développement. Presses universitaires de France.

Robida, A. (1892). La vie électrique : le vingtième siècle. Librairie illustrée.

Saint-Arnaud, Y. (1989). Les petits groupes : participation et communication. Presses de l'Université de Montréal.

Stacey, E. et Visser, L. (2005). The history of distance education in Australia. Quarterly Review of Distance Education, 6(3), 253-259.

Vieille-Grosjean, H. et Di Patrizio, G. (2015). Apprendre à l'âge adulte : entre imitation et émancipation. Phronesis, 4(1), 40-50. https://doi.org/10.7202/1031203ar

Vygotski, L. S. (1985). Pensée et langage (F. Sève, trad.). Messidor. (Ouvrage original publié en 1934.) 